Case Report

\title{
Oropharyngeal Dysphagia with Aspiration as a Provoking Factor for COPD Exacerbation
}

\author{
Noor Sameh Darwich ${ }^{1,}$, Shamsuddin Chowdhry Pracha ${ }^{2}$, Natalie Ann Miller ${ }^{3}$ \\ ${ }^{1}$ Faculty of Medicine, University of Jordan, Amman, Jordan \\ ${ }^{2}$ Department of Medicine, Kettering Medical Center, Kettering, Ohio, USA \\ ${ }^{3}$ Department of Physical Medicine, Rehabilitation and Speech Therapy, Sycamore Hospital, Miamisburg, Ohio, USA
}

Email address:

nsdarwich@gmail.com (N. S. Darwich)

${ }^{*}$ Corresponding author

\section{To cite this article:}

Noor Sameh Darwich, Shamsuddin Chowdhry Pracha, Natalie Ann Miller. Oropharyngeal Dysphagia with Aspiration as a Provoking Factor for COPD Exacerbation. American Journal of Internal Medicine. Vol. 7, No. 1, 2019, pp. 12-14. doi: 10.11648/j.ajim.20190701.14

Received: February 6, 2019; Accepted: March 1, 2019; Published: March 26, 2019

\begin{abstract}
The most common provoking factors for chronic Obstructive Pulmonary Disease (COPD) exacerbation include viral and bacterial tracheobronchitis, pneumonia, and exposure to environmental irritants and air pollution. In many patients with COPD exacerbation, the underlying cause cannot be identified. In general, patients with COPD exacerbation get admitted to the hospital and treated with antibiotics, glucocorticoids and inhaled bronchodilators. Oropharyngeal dysphagia is an under-recognized provoking factor for COPD exacerbation. Patients with advanced COPD often have impaired coordination of respiration and deglutition which can lead to aspiration of liquids, food particles, and saliva into the airways. Aspiration events can lead to exacerbation of symptoms and cause further decline in lung function. We described a 69-year-old male with a history of COPD who presented with progressive dyspnea, productive cough and hypoxia which required intubation and mechanical ventilation. The patient underwent a bronchoscopy for airway inspection which showed pieces of meat in the right main bronchus which were removed. Reportedly, the patient was having difficulty swallowing solid food prior to admission to the hospital.
\end{abstract}

Keywords: Chronic Obstructive Pulmonary Disease (COPD), Swallowing Dysfunction, Oropharyngeal Dysphagia, Aspiration

\section{Introduction}

Swallowing dysfunction in patients with advanced COPD is a common finding [1]. Postprandial aspiration of food particles and more commonly liquids into the airways can lead to COPD exacerbation. In turn, during COPD exacerbation from all causes, oropharyngeal dysphagia can worsen [2]. Repeated aspiration can lead to serious sequela in COPD patients including pneumonia, infection, worsening lung function and even death [3]. We detail the case of a patient with COPD exacerbation due to swallowing dysfunction and aspiration of meat particle into the airways, requiring intubation and mechanical ventilation support. Aspiration was confirmed in this patient via bronchoscopy with removal of the food particles from the right main bronchus. Oropharyngeal aspiration should be in the differential diagnoses of patients with COPD exacerbations particularly in those with advanced COPD and frequent exacerbations without obvious provoking factors [4]. The patient should be evaluated by a speech-Language Pathologist to further assess swallowing function. If silent aspiration is suspected, an objective assessment such as a Video fluoroscopy should be performed.

\section{Case Report}

A 69-year-old male with history of COPD and frequent admissions to the hospital due to acute respiratory failure requiring intubation presented to the emergency room department with a two-day history of progressive dyspnea and productive cough. He was recently discharged from the Hospital after treatment for COPD exacerbation with IV 
glucocorticoids, antibiotics and inhaled bronchodilators. On arrival to the emergency department, he was found to be hypoxic and was placed on 5 Liters of oxygen via nasal cannula. On exam, he had bilateral wheezing. Chest x-ray (Figure 1) showed diffuse bilateral prominent interstitial lung markings, which were unchanged from a previous chest x-ray. The patient was later admitted to the hospital and started on IV glucocorticoid, doxycycline and aggressive pulmonary toileting with inhaled bronchodilators. In spite of these treatments, his work of breathing and hypoxia worsened, and he was placed on Bi-level intermittent positive pressure ventilation (BIPAP), eventually requiring intubation and mechanical ventilation. Upon further questioning of the patient's family, they reported that the patient was having difficulty swallowing solid food recently, and an event of choking on the steak was reported to have occurred the night before admission. Indeed, the patient reported to his family that he felt like a piece of meat was stuck in his throat. Bronchoscopy was performed for airway inspection, which revealed pieces of meat in the right main bronchus, which were removed with forceps (Figure 2). A follow-up bronchoscopy was done two days later which revealed some purulent secretions, however, did not show any endobronchial obstruction. The patient was weaned successfully from the ventilator and extubated. He was referred to the speech therapy team for a swallowing evaluation.

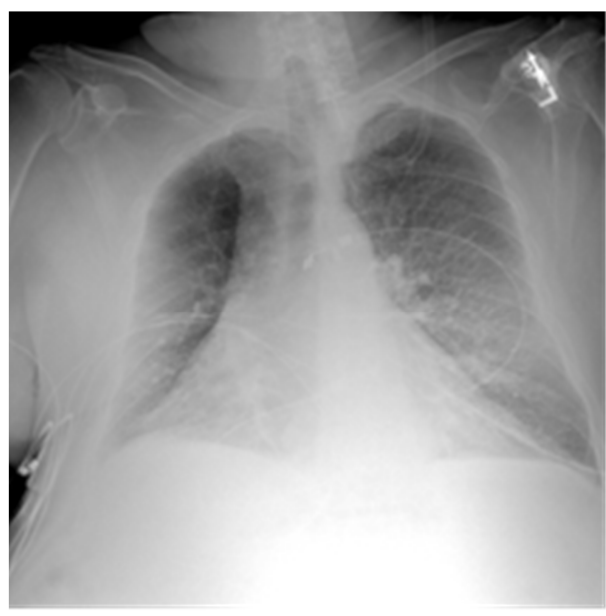

Figure 1. Chest radiography shows diffuse bilateral prominent interstitial lung markings.

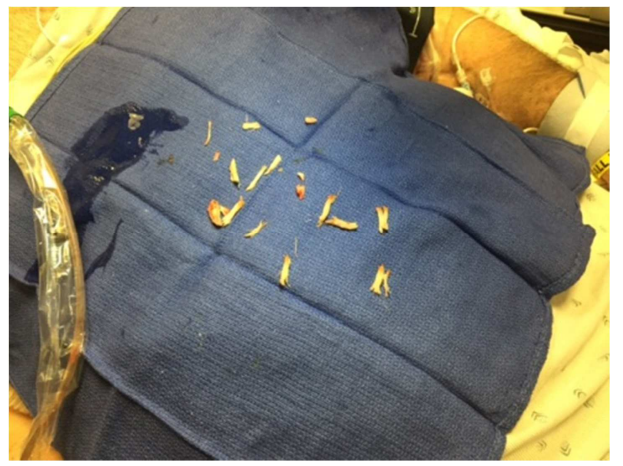

Figure 1. Pieces of meat removed from right main bronchus during bronchoscopy.

\section{Discussion}

Several studies have investigated the correlation between COPD exacerbation and aspiration [5]. Two recognized potential causes of aspiration include oropharyngeal dysphagia and Gastroesophageal Reflux Disease (GERD).

COPD patients have been shown to have increased incidence of swallowing dysfunction at their baseline as well as during an exacerbation [6]. Two common characteristics of swallowing dysfunction seen in COPD patients include disruption of the normal exhale-swallow-exhale pattern and an abnormal swallowing reflex [7]. Swallowing dysfunction patterns seen the most in patients with COPD include exhale-swallow-inhale and, inhale-swallow-inhale, swallowing during inhalation or swallowing during late exhalation. Repeated aspiration of oropharyngeal secretions leads to increased bacterial loads in distal airways which will increase the frequency of bacterial tracheobronchitis and pneumonia in patients with COPD. Studies have shown that there is a significant increase in hospitalization, morbidity, and mortality due to dysphagia in patients with COPD [8]. Swallowing dysfunction with silent aspiration is well known as a factor for COPD exacerbation.

Conversely, exacerbation of COPD may promote swallowing dysfunction and aspiration which in turn may increase the severity of COPD and frequency of exacerbations. Patients with experiencing exacerbation events have been found to have increase latency in the swallowing reflex, posing increasing aspiration risk, as compared to subjects that were not presently having exacerbation [9]. Dysphagia in individuals with COPD cannot be identified on clinical evaluation by the presence of coughing, which can be an obvious sign of aspiration, as a cough can be chronically present in individuals diagnosed with COPD. As noted above, aspiration may be silent without any warning symptoms [10]. In one study involving male patients with the primary diagnosis of COPD revealed that up to $85 \%$ of these patients exhibited some degree of swallowing dysfunction and laryngeal penetrations or aspirations on a Videofluoroscopy study [11]. Videofluoroscopy is highly recommended for the evaluation of dysphagia in COPD patient and is the gold standard to diagnose dysphagia and the presence of aspiration, especially silent aspiration as this cannot be ruled out by bedside assessment alone. Gastroesophageal Reflux Disease (GERD) is another common cause of microaspiration in the COPD patient [12]. Based on numerous studies, the prevalence of GERD in COPD patients ranges anywhere from $17 \%$ to $54 \%$. One study showed that pepsin was present in $33 \%$ of the sputum samples gathered from moderate to severe COPD patients [13]. Finally, tracheostomy tube; when indicated for patients with COPD; poses a high risk for swallowing dysfunction and aspiration as it interferes with normal swallowing [14]. 


\section{Conclusion}

Keeping a high clinical suspicion of swallowing dysfunction with aspiration in COPD patients with frequent exacerbations is important. The risk of aspiration secondary to oropharyngea dysphagia and GERD needs to be recognized early and managed appropriately in patients with COPD to prevent further exacerbation with declining lung function and to improve quality of life. Silent aspiration should be suspected and investigated through evaluation by speech therapy specialist. Videofluoroscopy is the gold standard for assessment of swallowing function in patients suffering from COPD.

\section{Conflict of Interest}

The authors declare no conflict of interest.

\section{References}

[1] Tsuzuki A, Kagaya H, Takahashi H, Shioya T, Sakakibara H, et al. Dysphagia causes exacerbation in individuals with chronic obstructive pulmonary disease. J Am Geriatric Soc 2012; 60 (8): $1580-1582$.

[2] O'Kane L, Groher M, Oropharyngeal Dysphagia in Patient with and obstructive pulmonary disease: a Systematic review. Rev CEFAC 2009; 11 (3): 499-506.

[3] Steidl E, Ribeiro CS, Goncalves BF, Fernandes N, Antunes V, Mancopes R. Relationship between Dysphagia and exacerbation in chronic Obstructive Disease. A literature review. International Archives of Otorhinolaryngology. Vol 19 , Page 74-79. No 1/ 2015.

[4] Donaldson GC, Wedzicha JA, COPD exacerbations. 1: Epidemology. Thorax 2006; 61: 164-8.
[5] Terada K, Muro S, Ohara T, Kudo M, Ogawa E, Hoshino Y and Hirai T, Niimi A, Chin K, Mishima M. Abnormal swallowing reflex and COPD exacerbation. Chest, 137 (2), 326-332. 2009.

[6] Mokhlesi B, Logemann JA, Rademaker AW, et al. oropharyngeal deglutition in stable COPD. Chest 2002; 121: 361-9.

[7] Stroud, Jayma. "Disordered Coordination of Deglutition and Respiration in Chronic Obstruction Pulmonary Disease." Research Papers (2014): Paper 480.

[8] Gross R, Atwood C, Ross S, Olszewski J, and Eichorn K, The coordination of breathing and swallowing in chronic obstruction pulmonary disease. American Journal of respiratory and Critical care Medicine, Vol 179, 559-565, 2009.

[9] Kobayashi, S., Kubo, H., Yanai, M. (2007). Impairment of the swallowing reflex in exacerbations of COPD. BMJ Journals, $62(11)$.

[10] Cvejic, L., Harding, R. Churchward A, Turton A., Finlay P., Massey D. et al. (2011). Laryngeal Penetration and aspiration in individuals with stable COPD. Respirology, Asian Pacific Society of Respirology. 16, 269-275. 2011.

[11] Good-Fratturelli, M. D., Curlee, R. F., Holle, J. L. Prevalence and nature of dysphagia in VA patients with COPD referred for Videofluoroscopic swallow examination. (2000) Journal of Communication Disorders, 33 (2), 93-110.

[12] Lee, Annemarie, and Roger Goldstein. "Gastroesphageal Reflux Disease in COPD: Links and Risks." International Journal of COPD (2015): 1935-949.

[13] Terada, K., S. Muro, S. Sato, T. Ohara, and A. Haruna. "Impact of Gastro-oesophageal Reflux Disease Symptoms on COPD Exacerbation." BMJ 63 (2008): 951-55.

[14] Coelho, C. Preliminary findings on the nature of dysphagia in patients with chronic obstructive pulmonary disease. Dysphagia 1987; 2 (1): 28-31. 